\title{
Genus, Treewidth, and Local Crossing Number
}

\author{
Vida Dujmović* $\quad$ David Eppstein ${ }^{\dagger} \quad$ David R. Wood ${ }^{\ddagger}$
}

June 16, 2015

\begin{abstract}
We consider relations between the size, treewidth, and local crossing number (maximum crossings per edge) of graphs embedded on topological surfaces. We show that an $n$-vertex graph embedded on a surface of genus $g$ with at most $k$ crossings per edge has treewidth $O(\sqrt{g k n})$ and layered treewidth $O(g k)$, and that these bounds are tight up to a constant factor. As a special case, the $k$-planar graphs with $n$ vertices have treewidth $O(\sqrt{k n})$ and layered treewidth $O(k)$, which are tight bounds that improve a previously known $O\left(k^{3 / 4} n^{1 / 2}\right)$ treewidth bound. Additionally, we show that for $g<m$, every $m$-edge graph can be embedded on a surface of genus $g$ with $O\left((\mathrm{~m} / \mathrm{g}) \log ^{2} g\right)$ crossings per edge, which is tight to within a polylogarithmic factor.
\end{abstract}

\section{Introduction}

Treewidth is a graph parameter that measures how similar a graph is to a tree. It is a key measure of the complexity of a graph and is of fundamental importance in algorithmic graph theory and structural graph theory, especially in Robertson and Seymour's graph minors project. Treewidth is closely related to the size of a smallest separator, a set of vertices whose removal splits the graph into connected components of at most $\frac{2 n}{3}$ vertices, where $n$ (as always) is the number of vertices in the graph. Graphs of low treewidth necessarily have small separators, and graphs in which every subgraph has a small separator have low treewidth $[2,11]$. See Section 2 for a detailed definition of treewidth.

A graph is $k$-planar if it can be drawn in the plane with at most $k$ crossings on each edge. The local crossing number of the graph is the minimum $k$ for which it is $k$-planar [13, pages 51-53]. An important example is the $p \times q \times r$ grid graph, with

\footnotetext{
${ }^{*}$ School of Computer Science and Electrical Engineering, University of Ottawa, Ottawa, Canada (vida.dujmovic@uottawa.ca)

${ }^{\dagger}$ Department of Computer Science, University of California, Irvine, California, USA (eppstein@uci.edu)

${ }^{\ddagger}$ School of Mathematical Sciences, Monash University, Melbourne, Australia (david. wood@monash.edu)
} 

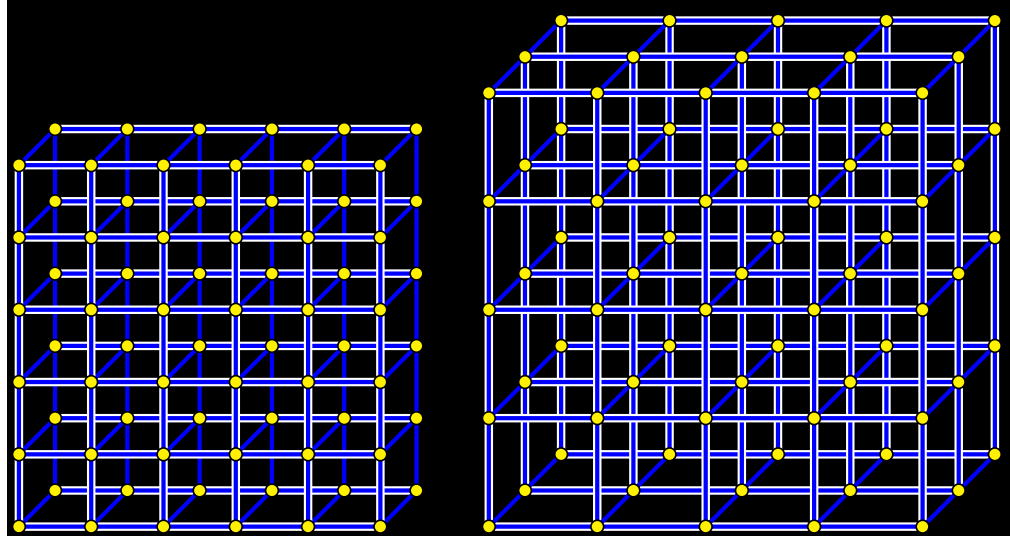

Figure 1: The $p \times q \times r$ grid graph is $(r-1)$-planar.

vertex set $[p] \times[q] \times[r]$ and all edges of the form $(x, y, z)(x+1, y, z)$ or $(x, y, z)(x, y+1, z)$ or $(x, y, z)(x, y, z+1)$. A suitable linear projection from the natural three-dimensional embedding of this graph to the plane gives a $(r-1)$-planar drawing, as illustrated in Figure 1.

The starting point for our work is the following question: what is the maximum treewidth of $k$-planar graphs? Grigoriev and Bodlaender [5] studied this question and proved an upper bound of $O\left(k^{3 / 4} n^{1 / 2}\right)$. We improve this and give the following tight bound:

Theorem 1. The maximum treewidth of $k$-planar $n$-vertex graphs is

$$
\Theta(\min \{n, \sqrt{(k+1) n}\}) .
$$

More generally, define a graph to be $(g, k)$-planar if it can be drawn in a surface of Euler genus at most $g$ with at most $k$ crossings on each edge. ${ }^{1}$ For instance, Guy et al. [7] investigated the local crossing number of toroidal embeddings - in this notation, the $(2, k)$-planar graphs. We again determine an optimal bound on the treewidth of such graphs.

Theorem 2. The maximum treewidth of $(g, k)$-planar $n$-vertex graphs is

$$
\Theta(\min \{n, \sqrt{(g+1)(k+1) n}\}) .
$$

In both these theorems, the $k=0$ case (with no crossings) is well known [4]. We prove our upper bounds by using the concept of layered treewidth [1], and we prove matching lower bounds by finding $(g, k)$-planar graphs without large separators and using the known relations between separator size and treewidth.

\footnotetext{
${ }^{1}$ The Euler genus is $2 h$ for an orientable surface with $h$ handles, and $c$ for a non-orientable surface with $c$ cross-caps.
} 
Finally, we study the $(g, k)$-planarity of graphs as a function of their number of edges. For (global) crossing number, a graph with $m$ edges drawn on a surface of genus $g$ may require $\Omega\left(\min \left\{m^{2} / g, m^{2} / n\right\}\right)$ crossings, and it can be drawn with $O\left(\left(m^{2} \log ^{2} g\right) / g\right)$ crossings [14]. In particular, the lower bound implies that some graphs require $\Omega(\mathrm{m} / \mathrm{g})$ crossings per edge on average, and therefore also in the worst case. We prove a nearlymatching upper bound:

Theorem 3. For every graph $G$ with $m$ edges, for every integer $g \geqslant 1$, there is a drawing of $G$ in the orientable surface with at most $g$ handles and with

$$
O\left(\frac{m \log ^{2} g}{g}\right)
$$

crossings per edge.

\section{Background}

For $\epsilon \in(0,1)$, a set $S$ of vertices in a graph $G$ is an $\epsilon$-separator of $G$ if each component of $G-S$ has at most $\epsilon|V(G)|$ vertices. It is conventional to set $\epsilon=2 / 3$ but the precise choice makes no difference to the asymptotic size of a separator.

A tree-decomposition of a graph $G$ is given by a tree $T$ whose nodes index a collection $\left(B_{x} \subseteq V(G): x \in V(T)\right)$ of sets of vertices in $G$ called bags, such that:

- For every edge $v w$ of $G$, some bag $B_{x}$ contains both $v$ and $w$, and

- For every vertex $v$ of $G$, the set $\left\{x \in V(T): v \in B_{x}\right\}$ induces a non-empty (connected) subtree of $T$.

The width of a tree-decomposition is $\max _{x}\left|B_{x}\right|-1$, and the treewidth $\operatorname{tw}(G)$ of a graph $G$ is the minimum width of any tree decomposition of $G$. Treewidth was introduced (with a different but equivalent definition) by Halin [8] and tree decompositions were introduced by Robertson and Seymour [12] who proved:

Lemma 4 ([12]). Every graph with treewidth $k$ has a $\frac{1}{2}$-separator of size at most $k+1$.

The notion of layered tree decompositions is a key tool in proving our main theorems. A layering of a graph $G$ is a partition $\left(V_{0}, V_{1}, \ldots, V_{t}\right)$ of $V(G)$ such that for every edge $v w \in E(G)$, if $v \in V_{i}$ and $w \in V_{j}$, then $|i-j| \leqslant 1$. Each set $V_{i}$ is called a layer. For example, for a vertex $r$ of a connected graph $G$, if $V_{i}$ is the set of vertices at distance $i$ from $r$, then $\left(V_{0}, V_{1}, \ldots\right)$ is a layering of $G$, called the bfs layering of $G$ starting from $r$. A $b$ s tree of $G$ rooted at $r$ is a spanning tree of $G$ such that for every vertex $v$ of $G$, the distance between $v$ and $r$ in $G$ equals the distance between $v$ and $r$ in $T$. Thus, if $v \in V_{i}$ then the $v r$-path in $T$ contains exactly one vertex from layer $V_{j}$ for $0 \leqslant j \leqslant i$.

The layered width of a tree-decomposition $\left(B_{x}: x \in V(T)\right)$ of a graph $G$ is the minimum integer $\ell$ such that, for some layering $\left(V_{0}, V_{1}, \ldots, V_{t}\right)$ of $G$, each bag $B_{x}$ contains at most $\ell$ vertices in each layer $V_{i}$. The layered treewidth of a graph $G$ is 
the minimum layered width of a tree-decomposition of $G$. Note that if we only consider the trivial layering in which every vertex is in a single layer, then layered treewidth equals treewidth plus 1 .

Dujmović, Morin, and Wood [1] introduced layered treewidth and proved the following results, where a graph $G$ is apex if $G-v$ is planar for some vertex $v$ :

Theorem 5 (Dujmović, Morin, and Wood [1]). (a) Every planar graph has layered treewidth at most 3 .

(b) Every graph with Euler genus $g$ has layered treewidth at most $2 g+3$.

(c) For every apex graph $H$, there is a number c such that every $H$-minor-free graph has layered treewidth at most $c$.

(d) If a minor-closed class has bounded layered treewidth, then it excludes a fixed apex graph as a minor.

The same characterization by forbidden apex minors was previously known for

minor-closed classes of bounded local treewidth [3], establishing the equivalence of bounded local treewidth and bounded layered treewidth in minor-closed classes; however, for families of graphs that are not minor-closed, layered treewidth and local treewidth are distinct. Sergey Norin established the following connection between layered treewidth and treewidth:

Theorem 6 (Norin; see [1]). Every $n$-vertex graph with layered treewidth $k$ has treewidth at most $2 \sqrt{k n}$.

Several results that follow depend on expanders; see [9] for a survey.

Lemma 7. For $k \geqslant 3$ and $n \geqslant k+1$ (such that $n$ is even if $k$ is odd), there is a $k$-regular $n$-vertex graph $H$ (called an expander) such that for every $\epsilon \in(0,1)$ there exists $\beta>0$, such that every $\epsilon$-separator in $H$ has size at least $\beta n$.

\section{$3 \quad k$-Planar Graphs}

The following is our first contribution.

Theorem 8. Every $k$-planar graph has layered treewidth at most $6(k+1)$.

Proof. Let $G$ be $k$-planar; draw $G$ with at most $k$ crossings per edge, and arbitrarily orient each edge of $G$. Let $G^{\prime}$ be the graph obtained from $G$ by replacing each crossing by a new degree-4 vertex. Then $G^{\prime}$ is planar. By Theorem $5(\mathrm{a}), G^{\prime}$ has layered treewidth at most 3. That is, there is a tree decomposition $T^{\prime}$ of $G^{\prime}$, and a layering $V_{0}^{\prime}, V_{1}^{\prime}, \ldots$ of $G^{\prime}$, such that each bag of $T^{\prime}$ contains at most three vertices in each layer $V_{i}^{\prime}$. For each vertex $v$ of $G^{\prime}$, let $T_{v}^{\prime}$ be the subtree of $T^{\prime}$ formed by the bags that contain $v$.

Let $T$ be the decomposition of $G$ obtained by replacing each occurrence of a dummy vertex $x$ in a bag of $T^{\prime}$ by the tails of the two edges that cross at $x$. We now show that 
$T$ is a tree-decomposition of $G$. For each vertex $v$ of $G$, let $T_{v}$ be the subgraph of $T$ formed by the bags that contain $v$. Let $G_{v}^{\prime}$ be the subgraph of $G^{\prime}$ induced by $v$ and the division vertices on the edges for which $v$ is the tail. Then $G_{v}^{\prime}$ is connected. Thus $T_{v}^{\prime}$, which is precisely the set of bags of $T^{\prime}$ that intersect $G_{v}^{\prime}$, form a (connected) subtree of $T^{\prime}$. Moreover, for each oriented edge $v w$ of $G$, if $x$ is the division vertex of $v w$ adjacent to $w$, then $T_{x}^{\prime}$ and $T_{w}^{\prime}$ intersect. Since $T_{v}$ contains $T_{x}^{\prime}$, and $T_{w}$ contains $T_{w}^{\prime}$, we have that $T_{v}$ and $T_{w}$ intersect. Thus $T$ is a tree-decomposition of $G$. By construction, each bag of $T$ contains at most six vertices in each layer $V_{i}^{\prime}$.

Note that $\operatorname{dist}_{G^{\prime}}(v, w) \leqslant k+1$ for each edge $v w$ of $G$. Thus, if $v \in V_{i}^{\prime}$ and $w \in V_{j}^{\prime}$ then $|i-j| \leqslant k+1$. Let $V_{0}$ be the union of the first $k+1$ layers restricted to $V(G)$, let $V_{1}$ be the union of the second $k+1$ layers restricted to $V(G)$, and so on. That is, for $i \geqslant 0$, let $V_{i}:=V(G) \cap\left(V_{(k+1) i}^{\prime} \cup V_{(k+1) i+1}^{\prime} \cup \cdots \cup V_{(k+1)(i+1)-1}\right)$. Then $V_{0}, V_{1}, \ldots$ is a partition of $V(G)$. Moreover, if $v \in V_{i}$ and $w \in V_{j}$ for some edge $v w$ of $G$, then $|i-j| \leqslant 1$. Thus $V_{1}, V_{2}, \ldots$ is a layering of $G$. Since each layer in $G$ consists of at most $k+1$ layers in $G^{\prime}$, the layered treewidth of this decomposition is at most $6(k+1)$.

Theorem 5 does not imply Theorem 8, because 1-planar graphs may contain arbitrarily large complete graph minors. For example, the $n \times n \times 2$ grid graph is 1-planar, and contracting the $i$-th row in the front grid with the $i$-th column in the back grid gives a $K_{n}$ minor.

Theorem 6 and Theorem 8 imply the upper bound in Theorem 1:

Theorem 9. Every $k$-planar $n$-vertex graph has treewidth at most $2 \sqrt{6(k+1) n}$.

We now prove the corresponding lower bound.

Theorem 10. For $1 \leqslant k \leqslant \frac{3}{2} n$ there is a $k$-planar graph on $n$ vertices with treewidth at least $c \sqrt{k n}$ for some constant $c>0$.

Proof. Let $G$ be a cubic expander with $n$ vertices. Then $G$ has treewidth at least $\epsilon n$ for some constant $\epsilon>0$ (see for example Grohe and Marx [6]). Consider a straight-line drawing of $G$. Clearly, each edge is crossed less than $|E(G)|=\frac{3}{2} n$ times. Subdivide each edge of $G$ at most $\frac{3 n}{2 k}$ times to produce a $k$-planar graph $G^{\prime}$ with $n^{\prime}$ vertices, where $n^{\prime} \leqslant n+\frac{3 n}{2} \frac{3 n}{2 k}<\frac{4 n^{2}}{k}$. Subdivision does not change the treewidth of a graph. Thus $G^{\prime}$ has treewidth at least $\epsilon n \geqslant \frac{\epsilon}{2} \sqrt{k n^{\prime}}$.

Combining the bound of Theorem 9 with the trivial upper bound $\operatorname{tw}(G) \leqslant n$ for $k \geq$ $n$ shows that the maximum treewidth of $k$-planar $n$-vertex graphs is $\Theta(\min \{n, \sqrt{k n}\})$ for arbitrary $k$ and $n$. This completes the proof of Theorem 1 .

\section{$4(g, k)$-Planar Graphs}

Recall that a graph is $(g, k)$-planar if can be drawn in a surface of Euler genus at most $g$ with at most $k$ crossings on each edge. The proof method used in Theorem 8 in conjunction with Theorem 5(b) leads to the following theorem. 
Theorem 11. Every $(g, k)$-planar graph $G$ has layered treewidth at most $(4 g+6)(k+1)$.

Proof. Consider a drawing of $G$ with at most $k$ crossings per edge on a surface $\Sigma$ of Euler genus $g$. Arbitrarily orient each edge of $G$. Let $G^{\prime}$ be the graph obtained from $G$ by replacing each crossing by a new degree- 4 vertex. Then $G^{\prime}$ is embedded in $\Sigma$ with no crossings, and thus has Euler genus at most $g$. By Theorem 5(b), $G^{\prime}$ has layered treewidth at most $2 g+3$. That is, there is a tree decomposition $T^{\prime}$ of $G^{\prime}$, and a layering $V_{0}^{\prime}, V_{1}^{\prime}, \ldots$ of $G^{\prime}$, such that each bag of $T^{\prime}$ contains at most $2 g+3$ vertices in each layer $V_{i}^{\prime}$. For each vertex $v$ of $G^{\prime}$, let $T_{v}^{\prime}$ be the subtree of $T^{\prime}$ formed by the bags that contain $v$.

Let $T$ be the decomposition of $G$ obtained by replacing each occurrence of a dummy vertex $x$ in a bag of $T^{\prime}$ by the tails of the two edges that cross at $x$. We now show that $T$ is a tree-decomposition of $G$. For each vertex $v$ of $G$, let $T_{v}$ be the subgraph of $T$ formed by the bags that contain $v$. Let $G_{v}^{\prime}$ be the subgraph of $G^{\prime}$ induced by $v$ and the division vertices on the edges for which $v$ is the tail. Then $G_{v}^{\prime}$ is connected. Thus $T_{v}^{\prime}$, which is precisely the set of bags of $T^{\prime}$ that intersect $G_{v}^{\prime}$, form a (connected) subtree of $T^{\prime}$. Moreover, for each oriented edge $v w$ of $G$, if $x$ is the division vertex of $v w$ adjacent to $w$, then $T_{x}^{\prime}$ and $T_{w}^{\prime}$ intersect. Since $T_{v}$ contains $T_{x}^{\prime}$, and $T_{w}$ contains $T_{w}^{\prime}$, we have that $T_{v}$ and $T_{w}$ intersect. Thus $T$ is a tree-decomposition of $G$. By construction, each bag of $T$ contains at most $4 g+6$ vertices in each layer $V_{i}^{\prime}$.

Note that $\operatorname{dist}_{G^{\prime}}(v, w) \leqslant k+1$ for each edge $v w$ of $G$. Thus, if $v \in V_{i}^{\prime}$ and $w \in V_{j}^{\prime}$ then $|i-j| \leqslant k+1$. Let $V_{0}$ be the union of the first $k+1$ layers restricted to $V(G)$, let $V_{1}$ be the union of the second $k+1$ layers restricted to $V(G)$, and so on. That is, for $i \geqslant 0$, let $V_{i}:=V(G) \cap\left(V_{(k+1) i}^{\prime} \cup V_{(k+1) i+1}^{\prime} \cup \cdots \cup V_{(k+1)(i+1)-1}\right)$. Then $V_{0}, V_{1}, \ldots$ is a partition of $V(G)$. Moreover, if $v \in V_{i}$ and $w \in V_{j}$ for some edge $v w$ of $G$, then $|i-j| \leqslant 1$. Thus $V_{1}, V_{2}, \ldots$ is a layering of $G$. Since each layer in $G$ consists of at most $k+1$ layers in $G^{\prime}$, the layered treewidth of this decomposition is at most $(4 g+6)(k+1)$.

Theorem 11 and Theorem 6 imply:

Theorem 12. Every $n$-vertex $(g, k)$-planar graph has treewidth at most

$$
2 \sqrt{(4 g+6)(k+1) n} .
$$

We now show that this bound is tight up to a constant factor.

Theorem 13. For all $g, k \geqslant 0$ and infinitely many $n$ there is an $n$-vertex $(g, k)$-planar graph with treewidth $\Omega(\sqrt{(g+1)(k+1) n}$.

The proof of this result depends on the separation properties of the $p \times q \times r$ grid graph (which is $(r-1)$-planar). The next two results are not optimal, but have simple proofs and are all that is needed for the main proof that follows.

Lemma 14. For $q \geqslant\left(\frac{1}{1-\epsilon}\right) r$, every $\epsilon$-separator of the $q \times r$ grid graph has size at least $r$. 
Proof. Let $S$ be a set of at most $r-1$ vertices in the $q \times r$ grid graph. Some row $R$ avoids $S$, and at least $q-r+1$ columns avoid $S$. The union of these columns with $R$ induces a connected subgraph with at least $(q-r+1) r>\epsilon q r$ vertices. Thus $S$ is not an $\epsilon$-separator.

Lemma 15. For $p \geqslant q \geqslant\left(\frac{1}{1-\epsilon}\right) r$, every $\epsilon$-separator of the $p \times q \times r$ grid graph has size at least $\left(\frac{1-\epsilon}{1+\epsilon}\right) q r$.

Proof. Let $G$ be the $p \times q \times r$ grid graph. Let $n:=|V(G)|=p q r$. Let $S$ be an $\epsilon$ separator of $G$. Let $A_{1}, \ldots, A_{c}$ be the components of $G-S$. Thus $\left|A_{i}\right| \leqslant \epsilon n$. For $x \in[p]$, let $G_{x}:=\{(x, y, z): y \in[q], z \in[r]\}$ called a slice. Say $G_{x}$ belongs to $A_{i}$ and $A_{i}$ owns $G_{x}$ if $\left|A_{i} \cap G_{x}\right| \geqslant \frac{1+\epsilon}{2} q r$. Clearly, no two components own the same slice. First suppose that at least two components each own a slice. That is, $G_{v}$ belongs to $A_{i}$ and $G_{w}$ belongs to $A_{j}$ for some $v<w$ and $i \neq j$. Let $X:=\{(y, z):(v, y, z) \in$ $\left.G_{v},(w, y, z) \in G_{w}\right\}$. Then $|X| \geqslant 2\left(\frac{1+\epsilon}{2}\right) q r-q r=\epsilon q r$. For each $(y, z) \in X$, the 'straight' path $(v, y, z)(v+1, y, z), \ldots,(w, y, z)$ contains some vertex in $S$. Since these paths are pairwise disjoint, $|S| \geqslant|X| \geqslant \epsilon q r \geqslant \frac{1-\epsilon}{1+\epsilon} q r$ (since $\epsilon>\frac{1}{2}$ ). Now assume that at most one component, say $A_{1}$, owns a slice. Say $A_{1}$ owns $t$ slices. Thus $t\left(\frac{1+\epsilon}{2}\right) q r \leqslant\left|A_{i}\right| \leqslant \epsilon p q r$ and $t \leqslant \frac{2 \epsilon}{1+\epsilon} p$. Hence, at least $\left(1-\frac{2 \epsilon}{1+\epsilon}\right) p$ slices belong to no component. For such a slice $G_{v}$, each component of $G_{v}-S$ is contained in some $A_{i}$ and thus has at most $\left(\frac{1+\epsilon}{2}\right) q r$ vertices. That is, $S \cap G_{v}$ is a $\left(\frac{1+\epsilon}{2}\right)$-separator of the $q \times r$ grid graph induced by $G_{v}$. By Lemma 14, $\left|S \cap G_{v}\right| \geqslant r$. Thus $|S| \geqslant\left(1-\frac{2 \epsilon}{1+\epsilon}\right) p r \geqslant\left(\frac{1-\epsilon}{1+\epsilon}\right) q r$.

Proof of Theorem 13. Let $r:=k+1$.

First suppose that $g \leqslant 19$. Let $G$ be the $q \times q \times r$ grid graph where $q \geqslant 2 r$. As observed above, $G$ is $k$-planar and thus $(g, k)$-planar. Lemma 15 implies that every $\frac{1}{2}$-separator of $G$ has size at least $\frac{1}{3} q r$. Lemma 4 thus implies that $G$ has treewidth at least $\frac{1}{3} q r-1$, which is at least $\Omega(\sqrt{(g+1)(k+1) n})$, as desired.

Now assume that $g \geqslant 20$. By Lemma 7 there is a 4-regular expander $H$ on $m:=$ $\left\lfloor\frac{g}{4}\right\rfloor \geqslant 5$ vertices. Thus $H$ has $2 m$ edges, $H$ embeds in the orientable surface with $2 m$ handles, and thus has Euler genus at most $4 m \leqslant g$. We may assume that $q:=\sqrt{n / r m}$ is an integer with $q \geqslant 8 r$. Let $G$ be obtained from $H$ by replacing each vertex $v$ of $H$ by a copy of the $q \times q \times r$ grid graph with vertex set $D_{v}$, and replacing each edge of $H$ by a matching of $q r$ edges, so that $G\left[D_{v} \cup D_{w}\right]$ is a $2 q \times q \times r$ grid, as shown in Figure 2 . Thus $G$ is $(g, k)$-planar with $q^{2} r m=n$ vertices.

Let $S$ be a $\frac{1}{2}$-separator in $G$. Let $A_{1}, \ldots, A_{c}$ be the components of $G-S$. Thus $\left|A_{i}\right| \leqslant \frac{1}{2} n$ for $i \in[c]$. Initialise sets $S^{\prime}:=A_{1}^{\prime}:=\cdots:=A_{c}^{\prime}:=\emptyset$.

For each vertex $v$ of $H$, if $\left|S \cap D_{v}\right| \geqslant \frac{q r}{14}$ then put $v \in S^{\prime}$. Otherwise, $\left|S \cap D_{v}\right|<\frac{q r}{14}$. Note that Lemma 15 is applicable with $\epsilon=\frac{13}{15}$ since $q \geqslant 8 r>\frac{1}{1-13 / 15} r$ and $\frac{1-13 / 15}{1+13 / 15}=\frac{1}{14}$. Lemma 15 thus implies that $S \cap D_{v}$ is not a $\frac{13}{15}$-separator. Hence some component of $D_{v}-S$ has at least $\frac{13}{15} q^{2} r$ vertices. Since $\frac{13}{15}>\frac{1}{2}$, exactly one component of $D_{v}-S$ has at least $\frac{13}{15} q^{2} r$ vertices. This component is a subset of $A_{i}$ for some $i \in[c]$; add $v$ to $A_{i}^{\prime}$. Thus $S^{\prime}, A_{1}^{\prime} \ldots, A_{c}^{\prime}$ is a partition of $V(H)$. 


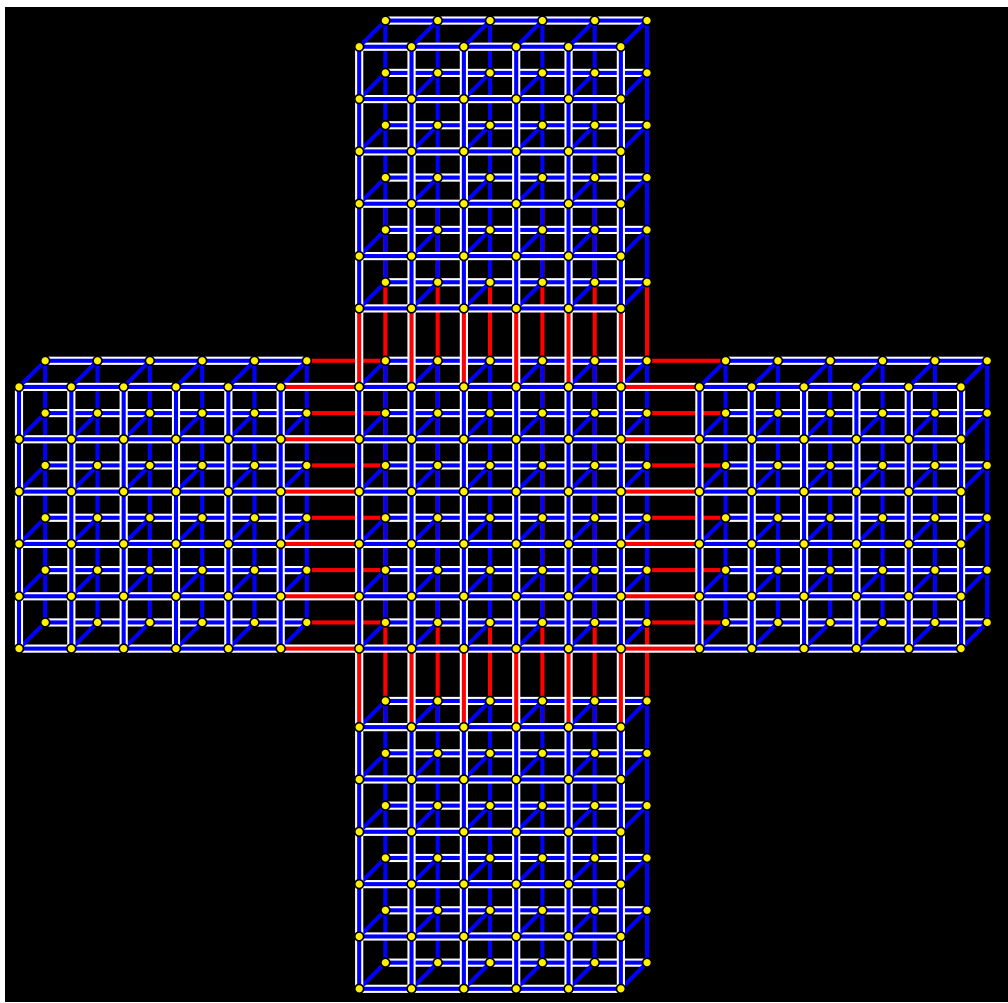

Figure 2: Construction of $G$ in the proof of Theorem 13.

We now prove that $S^{\prime}$ is a $\frac{15}{26}$-separator in $H$. Suppose that $v \in A_{i}^{\prime}$ and $w \in A_{j}^{\prime}$ for some edge $v w$ of $H$. Let $D$ be the vertex set of the $2 q \times q \times r$ grid graph induced by $D_{v} \cup D_{w}$. Since $v \notin S^{\prime}$ and $w \notin S^{\prime}$, we have $\left|S \cap D_{v}\right|<\frac{q r}{14}$ and $\left|S \cap D_{w}\right|<\frac{q r}{14}$. Thus $|S \cap D|<\frac{q r}{7}$. Note that Lemma 15 is applicable with $\epsilon=\frac{3}{4}$ since $q \geqslant 8 r>\frac{1}{1-3 / 4} r$ and $\frac{1-3 / 4}{1+3 / 4}=\frac{1}{7}$. Lemma 15 thus implies that $S \cap D$ is not a $\frac{3}{4}$-separator of $G[D]$. Hence some component $X$ of $G[D]-S$ contains at least $\frac{3}{4}|D|=\frac{3}{2} q^{2} r$ vertices. Each of $D_{v}$ and $D_{w}$ can contain at most $q^{2} r$ vertices in $X$. Thus $D_{v}$ and $D_{w}$ each contain at least $\frac{1}{2} q^{2} r$ vertices in $X$. Thus, by construction, $v$ and $w$ are in the same $A_{i}^{\prime}$. That is, there is no edge of $H$ between distinct $A_{i}^{\prime}$ and $A_{j}^{\prime}$, and each component of $H-S^{\prime}$ is contained in some $A_{i}^{\prime}$. For each $i \in[c]$, we have $\frac{1}{2} q^{2} r m \geqslant\left|A_{i}\right| \geqslant \frac{13}{15} q^{2} r\left|A_{i}^{\prime}\right|$ implying $\left|A_{i}^{\prime}\right| \leqslant \frac{15}{26} m$. Therefore $S^{\prime}$ is a $\frac{15}{26}$-separator in $H$.

By Lemma 7, $\left|S^{\prime}\right| \geqslant \beta m$ for some constant $\beta>0$. Thus $|S| \geqslant \frac{q r}{14}\left|S^{\prime}\right| \geqslant \frac{\beta}{14} m q r$. By Lemma $4, G$ has treewidth at least $\frac{\beta}{14} m q r-1=\frac{\beta}{14} \sqrt{m r n}-1 \geqslant \Omega(\sqrt{g(k+1) n})$, as desired.

Note that the proof of Theorem 13 in the case $k=0$ is very similar to that of Gilbert, Hutchison, and Tarjan [4].

For $g k \geqslant n$ the trivial upper bound of $\operatorname{tw}(G) \leqslant n$ is better than that given in The- 
orem 12. We conclude that the maximum treewidth of $(g, k)$-planar $n$-vertex graphs is $\Theta(\min \{n, \sqrt{(g+1)(k+1) n}\})$ for arbitrary $g, k, n$. This completes the proof of Theorem 2.

\section{Drawings with Few Crossings per Edge}

This section studies the following natural conjecture: for every surface $\Sigma$ of Euler genus $g$, every graph $G$ with $m$ edges has a drawing in $\Sigma$ with $O\left(\frac{m}{g+1}\right)$ crossings per edge. This conjecture is trivial at both extremes: with $g=0$, every graph has a straight-line drawing in the plane (and therefore a drawing in the sphere) with at most $m$ crossings per edge, and with $g=2 m$, every graph has a drawing in the the orientable surface with one handle per edge. Moreover, if this conjecture is true, it would provide a simple proof of Theorem 13 in the same manner as the proof of Theorem 10.

Our starting point is the following well-known result of Leighton and Rao [10, Theorem 22, p. 822]:

Theorem 16 (Leighton and Rao [10]). Let $G$ be a graph with bounded degree and $n$ vertices, mapped one-to-one onto the vertices of an expander graph $H$. Then the edges of $G$ can be mapped onto paths in $H$ so that each path has length $O(\log n)$ and each edge of $H$ is used by $O(\log n)$ paths.

It is straightforward to extend this result to regular graphs $G$ of unbounded degree, with the number of paths per edge of $H$ increasing in proportion to the degree. However, there are two difficulties with using it in our application. First, it does not directly handle graphs in which there is considerable variation in degree from vertex to vertex: in such cases we would want the number of paths per edge to be controlled by the average degree in $G$, but instead it is controlled by the maximum degree. And second, it does not allow us to control separately the sizes of $G$ and $H$; instead, both must have the same number of vertices. To handle these issues, we do not map the vertices of our input graph $G$ directly to the vertices of an expander $H$; instead, we keep the vertices of $G$ and the vertices of $H$ disjoint from each other, connecting them by a bipartite graph that balances the degrees, according to the following lemma.

Lemma 17. Let $d_{1}, d_{2}, \ldots, d_{n}$ be a sequence of positive integers, and let $q$ be any positive integer. Then there exists a bipartite graph with colour classes $\left\{v_{1}, \ldots, v_{n}\right\}$ and $\left\{w_{1}, \ldots, w_{q}\right\}$, at most $n+q-1$ edges, and a labeling of the edges with positive integers, such that

- each vertex $v_{i}$ is incident to a set of edges whose labels sum to $d_{i}$, and

- each pair of distinct vertices $w_{i}$ and $w_{j}$ are incident to sets of edges whose label sums differ by at most 1 .

Proof. Preassign label sums of $\left\lfloor\sum d_{i} / q\right\rfloor$ or $\left\lceil\sum d_{i} / q\right\rceil$ to each vertex $w_{i}$ so that the resulting values sum to $\sum d_{i}$. We will construct a bipartite graph and a labeling whose 

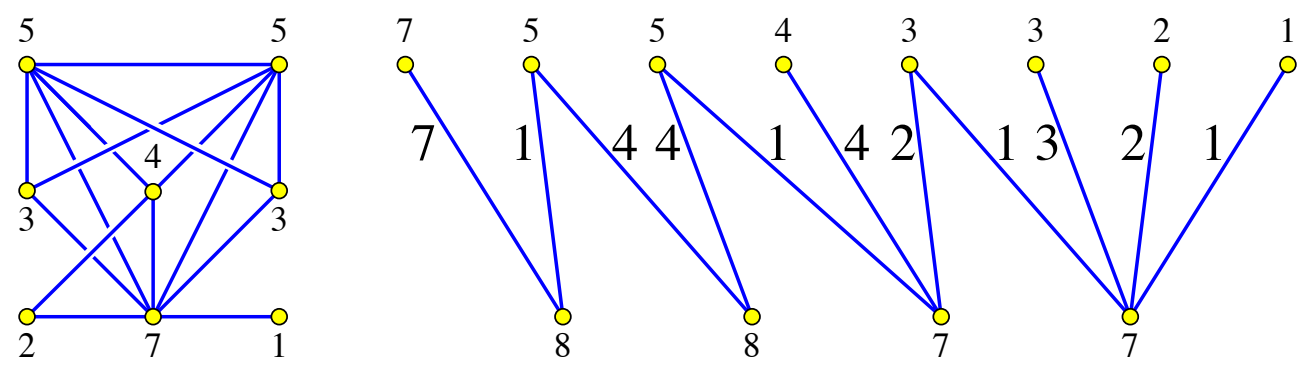

Figure 3: A graph (left) with degree sequence 7,5, 5, 4,3,3,2,1 and a bipartite graph (right) formed from this degree sequence by Lemma 17. The large numbers are the edge labels of the lemma, and the small numbers along the top and bottom of the bipartite graph give the sums of incident edge labels at each vertex. The top sums match the given degree sequence, while the bottom sums all differ by at most 1 .

sums match the numbers $d_{1}, \ldots, d_{n}$ on one side of the bipartition and whose sums match the preassigned numbers on the other side.

Build this graph and its labeling one edge at a time, starting from a graph with no edges. At each step, let $v_{i}$ and $w_{j}$ be the vertices on each side of the bipartition with the smallest indices whose edge labels do not yet sum to the required values, add an edge from $v_{i}$ to $w_{j}$, and label this edge with the largest integer that does not exceed the required sum on either vertex.

Each such step completes the sum for at least one vertex. Because the required values on the two sides of the bipartition both sum to $\sum d_{i}$, the final step completes the sum for two vertices, $v_{n}$ and $w_{q}$. Therefore, the total number of steps, and the total number of edges added to the graph, is at most $n+q-1$.

By combining this load-balancing step with the Leighton-Rao expander-routing scheme, we may obtain a more versatile mapping of our given graph $G$ to a host graph $H$, with better control over the genus of the surface we obtain from $H$. This genus will be determined by the cyclomatic number of $H$, where the cyclomatic number of a graph with $n$ vertices and $m$ edges is $m-n+1$. This number is the dimension of the cycle space of the graph, and the first Betti number of the topological space obtained from the graph by replacing each edge by a line segment.

Lemma 18. Let $G$ be an arbitrary graph, with $m$ edges, and let $Q$ be a q-vertex boundeddegree expander graph. Then there exists a host graph $H$, a one-to-one mapping of the vertices of $G$ to a subset of vertices of $H$, and a mapping of the edges of $G$ to paths in $H$, with the following properties:

- The vertices of $H$ that are not images of vertices in $G$ induce a subgraph isomorphic to $Q$.

- The image of an edge e in $G$ forms a path of length $O(\log q)$ that starts and ends at the image of the endpoints of $e$, and passes through the image of no other vertex of $G$. 
- Each vertex of $H$ that is not an image of a vertex in $G$ is crossed by $O((m \log q) / q)$ paths.

- The cyclomatic number of $H$ is $O(q)$.

Proof. Let the vertices of $G$ be $u_{1}, \ldots, u_{n}$. Apply Lemma 17 to the degree sequence of $G$ to form a bipartite graph with bipartition $\left\{v_{1}, \ldots, v_{n}\right\},\left\{w_{1}, \ldots, q_{q}\right\}$. Then add edges between pairs of vertices $\left(w_{i}, w_{j}\right)$ so that $\left\{w_{1}, \ldots, w_{q}\right\}$ induces a subgraph isomorphic to $Q$. In this way, each vertex $u_{i}$ in $G$ is mapped to a vertex $v_{i}$ in $H$ so that the mapping is one-to-one and the unmapped vertices form a copy of $Q$, as required. The cyclomatic number of $H$ equals the cyclomatic number of $Q$, plus $n+q-1$ (for the added edges in the bipartite graph), minus $n$ (for the added vertices relative to $Q$ ). These two added and subtracted terms cancel, leaving the cyclomatic number of $Q$ plus $q-1$, which is $O(q)$ as required.

It remains to find paths in $H$ corresponding to the edges in $G$. Assign each edge $u_{i} u_{j}$ of $G$ to a pair of vertices $\left(w_{i^{\prime}}, w_{j^{\prime}}\right)$ adjacent to the images $v_{i}$ and $v_{j}$ in $H$, so that the number of edges of $G$ assigned to each edge between $\left\{v_{1}, \ldots, v_{n}\right\}$ and $\left\{w_{1}, \ldots, w_{q}\right\}$ equals the corresponding label. Complete each path by applying Theorem 16 to the copy of $Q$; this gives paths of length $O(\log q)$ connecting each pair $\left(w_{i^{\prime}}, w_{j^{\prime}}\right)$ obtained in this way. These pairs do not form a bounded-degree graph, but they can be partitioned into $O(\mathrm{~m} / \mathrm{q})$ bounded-degree graphs, each of which causes each vertex in the copy of $Q$ to be crossed $O(\log q)$ times. Combining these suproblems, each vertex in the copy of $Q$ is crossed by a total of $O((m \log q) / q)$ paths, as required.

We are now ready to prove the existence of embeddings with small local crossing number, on surfaces of arbitrary genus.

Proof of Theorem 3. Given a graph $G$, to be embedded on a surface with at most $g$ handles and with few crossings per edge, choose $q$ so that the $O(q)$ bound on the cyclomatic number of the graph $H$ in Lemma 18 is at most $g$, and apply Lemma 18 to find a graph $H$ and a mapping from $G$ to $H$ obeying the conditions of the lemma.

To turn this mapping into the desired embedding of $G$, replace each vertex of degree $d$ in $H$ by a sphere, punctured by the removal of $d$ unit-radius disks, and form a surface (as an abstract topological complex, not necessarily embedded into three-dimensional space) by replacing each edge $x y$ of $H$ by a unit-radius cylinder connecting boundaries of removed disks on the spheres for vertices $x$ and $y$. The number of handles on the resulting surface (shown in Figure 4) equals the cyclomatic number of $H$. which is at most $g$.

Embed each vertex of $G$ as an arbitrarily chosen point on the sphere of the corresponding vertex of $H$, and each edge of $G$ as a curve through the sequence of spheres and cylinders corresponding to its path in $H$. Choose this embedding so that no intersection of edge curves occurs within any of the cylinders, and so that every pair of edges that are mapped to curves on the same sphere meet at most once, either at a crossing point or a shared endpoint. 


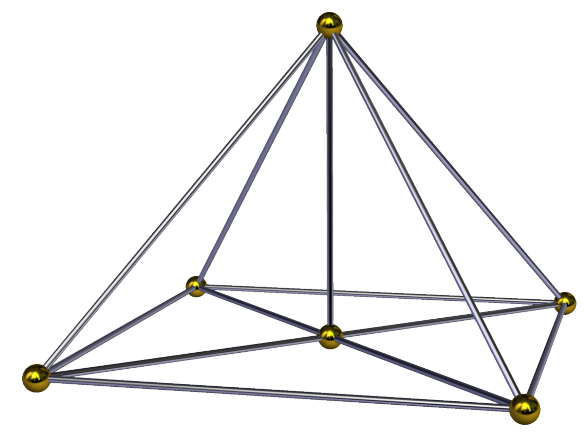

Figure 4: A topological surface obtained by replacing each vertex of a graph by a punctured sphere, and each edge of the graph by a cylinder connecting two punctures. Image Square_pyramid_pyramid.png by Tom Ruen on Wikimedia commons, made available under a Creative Commons CC-BY-SA 4.0 International license.

Because the spheres that contain vertices of $G$ only contain curves incident to those vertices, they do not have any crossings. Each edge is mapped to a curve through $O(\log g)$ of the remaining spheres, and can cross at most $O((m \log g) / g)$ other curves within each such sphere. Therefore, the maximum number of crossings per edge is $O\left(\left(m \log ^{2} g\right) / g\right)$.

Acknowledgement. This research was initiated at the Workshop on Graphs and Geometry held at the Bellairs Research Institute in 2015. Vida Dujmović was supported by NSERC. David Eppstein was supported in part by NSF grant CCF-1228639. David Wood was supported by the Australian Research Council.

\section{References}

[1] Vida Dujmović, Pat Morin, and David R. Wood, Layered separators in minor-closed families with applications, Electronic preprint arXiv: 1306.1595, 2013.

[2] Zdenek Dvorák and Sergey Norin, Treewidth of graphs with balanced separations, Electronic preprint arXiv: 1408.3869, 2014.

[3] David Eppstein, Diameter and treewidth in minor-closed graph families, Algorithmica 27 (2000), 275-291, doi:10.1007/s004530010020, MR 1759751.

[4] John R. Gilbert, Joan P. Hutchinson, and Robert E. Tarjan, A separator theorem for graphs of bounded genus, J. Algorithms 5 (1984), no. 3, 391-407, doi:10.1016/ 0196-6774(84)90019-1, MR 0756165.

[5] Alexander Grigoriev and Hans L. Bodlaender, Algorithms for graphs embeddable with few crossings per edge, Algorithmica 49 (2007), no. 1, 1-11, doi:10.1007/ s00453-007-0010-x, MR 2344391. 
[6] Martin Grohe and Dániel Marx, On tree width, bramble size, and expansion, J. Combin. Theory Ser. B 99 (2009), no. 1, 218-228, doi:10.1016/j.jctb.2008. 06.004, MR 2467827.

[7] Richard K. Guy, Tom Jenkyns, and Jonathan Schaer, The toroidal crossing number of the complete graph, J. Combinatorial Theory 4 (1968), 376-390, doi:10.1016/ S0021-9800 (68)80063-8, MR 0220630.

[8] Rudolf Halin, S-functions for graphs, J. Geometry 8 (1976), no. 1-2, 171-186, doi:10.1007/BF01917434, MR 0444522.

[9] Shlomo Hoory, Nathan Linial, and Avi Wigderson, Expander graphs and their applications, Bull. Amer. Math. Soc. (N.S.) 43 (2006), no. 4, 439-561, doi:10. 1090/S0273-0979-06-01126-8, MR 2247919.

[10] Tom Leighton and Satish Rao, Multicommodity max-flow min-cut theorems and their use in designing approximation algorithms, J. ACM 46 (1999), no. 6, 787832, doi:10.1145/331524.331526, MR 1753034.

[11] Bruce A. Reed, Tree width and tangles: a new connectivity measure and some applications, Surveys in combinatorics, London Math. Soc. Lecture Note Ser., vol. 241, Cambridge Univ. Press, 1997, pp. 87-162, doi:10.1017/CB09780511662119. 006, MR 1477746.

[12] Neil Robertson and P. D. Seymour, Graph minors. II. Algorithmic aspects of tree-width, J. Algorithms 7 (1986), no. 3, 309-322, doi:10.1016/0196-6774(86) 90023-4, MR 855559.

[13] Marcus Schaefer, The graph crossing number and its variants: A survey, Electron. J. Combin. (2014), DS21, available from http://www.combinatorics.org/ojs/ index.php/eljc/article/view/DS21.

[14] F. Shahrokhi, L. A. Székely, O. Sýkora, and I. Vrt'o, Drawings of graphs on surfaces with few crossings, Algorithmica 16 (1996), no. 1, 118-131, doi:10.1007/ s004539900040, MR 1394497. 